\title{
ASYMPTOTIC APPROXIMATIONS OF FIRST INTEGRALS FOR A NONLINEAR OSCILLATOR
}

\author{
S. B. Waluya ${ }^{\dagger}$ and W.T. van Horssen \\ Department of Applied Mathematical Analysis, Faculty of Information Technology \\ and Systems, Delft University of Technology, Mekelweg 4, 2628 CD Delft, The \\ Netherlands \\ (stevanus@dv.twi.tudelft.nl, W.T.vanHorssen@math.tudelft.nl)
}

\begin{abstract}
In this paper a generalized Rayleigh oscillator will be studied. It will be shown that the recently developed perturbation method based on integrating factors can be used to approximate first integrals and periodic solutions. The existence, uniqueness and stability of time-periodic solutions are obtained by using the approximations for the first integrals.
\end{abstract}

Key words. Integrating factor, integrating vector, first integral, perturbation method, asymptotic approximation of first integral.

1. Introduction. The fundamental concept to reduce a first order ordinary differential equation to an exact one by means of integrating factors has been extended in [9] to systems of first order ordinary differential equations. In [10, 11] a perturbation method based on these integrating factors has been presented for regularly perturbed ordinary differential equations (ODEs). When approximations of integrating factors have been obtained an approximation of a first integral can be given. Also an errorestimate for this approximation of a first integral can be given on a time-scale. It has also been shown in [10] how the existence and stability of time-periodic solutions for weakly nonlinear problems can be obtained from these approximations for the first integrals. In this paper the recently developed perturbation method based on integrating factors is used to approximate first integrals and periodic solutions for a generalized, nonlinear Rayleigh oscillator of the form

$$
\ddot{Z}+Z+b Z^{2}=c\left(1-\frac{\dot{Z}^{2}}{3}\right) \dot{Z},
$$

where $Z=Z(t)$, and where $b$ and $c$ are constants. The dot represents differentiation with respect to $t$. In this paper we consider two cases: (i) $b=\mathcal{O}(\epsilon)$ and $c=\epsilon^{2}$, and (ii) $b=\mathcal{O}(1)$ and $c=\epsilon$, where $\epsilon$ is a small parameter satisfying $0<\epsilon<<1$. In this paper not only asymptotic approximations of first integrals are constructed but also asymptotic approximations of periodic solutions and their periods are determined. The presented results include existence, uniqueness, and stability properties of the periodic solutions. In [3] van der Beek uses (1.1) with $b=\mathcal{O}(\epsilon)$ and $c=\epsilon^{2}$ as mathematical model to describe flow-induced vibrations of an oscillator with one degree of freedom in a uniform windfield. The oscillator and the frame are shown in Figure 1.1. The cylinder with ridge is rigidly attached to two shafts. These two shafts can simultaneously move within two air-bearings in the $z$-direction. The springs provide the restoring forces in the $z$-direction. The constant windflow is in $y$-direction. The mathematical model that describes the flow-induced vibrations of the oscillator

\footnotetext{
$\dagger$ On leave from: Mathematics Department Semarang State University, Indonesia
} 


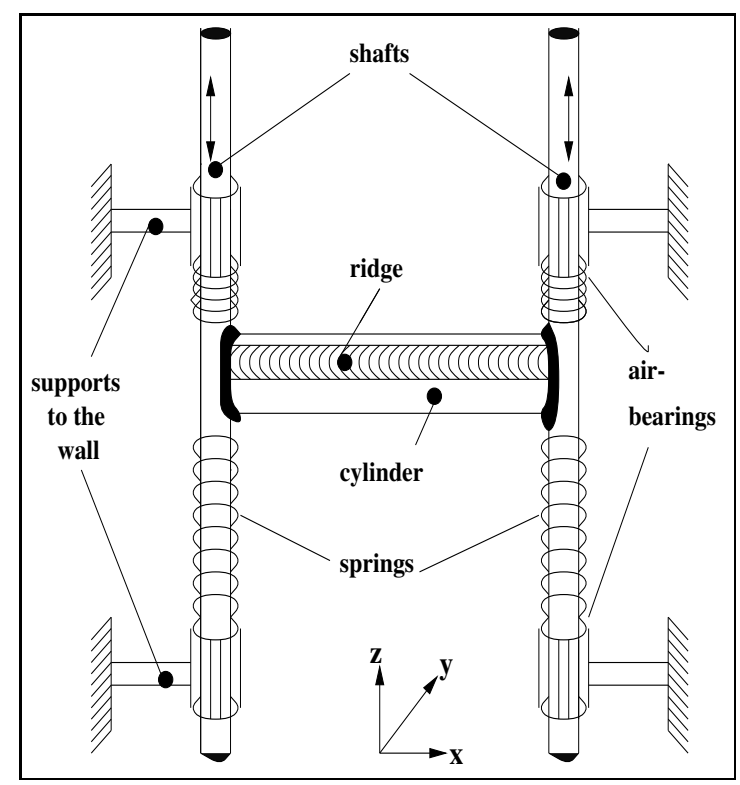

FIG. 1.1. The oscillator.

with one degree of freedom in a uniform wind-field can be given by

$$
\ddot{Z}+Z+\epsilon a Z^{2}=\epsilon^{2}\left(1-\frac{\dot{Z}^{2}}{3}\right) \dot{Z},
$$

where $\epsilon$ is a small parameter and where $a$ is a constant of order one. In [3] it has been shown how $\epsilon$ and $a$ depend on the physical quantities such as the windvelocity, the aerodynamic drag and lift forces acting on the cylinder, and so on. When the four springs are assumed to be nonlinear springs we can take (1.1) with $b=\mathcal{O}(1)$ and $c=\epsilon$ as a mathematical model to describe the flow-induced vibrations of the oscillator. Doelman and Verhulst [1], Wiggins [7], and many others used the Melnikov or Poincare-return map technique to study similar equations. In fact (1.1) has also been considered in [1] using the Poincare-return map technique. However, it has not been shown in [1] that (1.1) has at most two limit cycles. Using the perturbation method based on integrating vectors and some numerical calculations we will give strong numerical evidence that equation (1.1) has at most two limit cycles. Moreover, we will give in this paper explicit approximations of the periods of the periodic solutions. In this paper we show that straightforward expansions in $\epsilon$ can be used to construct asymptotic results on long time-scales. To obtain these results no classical perturbation techniques (such as averaging (see [2], [4]), or multiple (time) scales (see [6], [11]), or Melnikov/Poincare-return map techniques (see [1], [5], [7], [8])) are used. This paper is organized as follows. In section 2 of this paper the perturbation methods based on integrating factors and an asymptotic theory will be given briefly. It will be shown in section 3 of this paper how approximations of first integrals can be constructed. In section 4 it will be shown how the existence and stability of time-periodic solutions can be obtained. Finally in section 5 of this paper some conclusions will be drawn and some remarks will be made. 
2. Integrating factors and an asymptotic theory. In this section we briefly outline the perturbation method based on integrating factors as given in $[9,10,11]$. We consider the system of $n$ first order ODEs $(n \geq 1)$

$$
\frac{d \underline{y}}{d t}=\underline{f}(\underline{y}, t)
$$

where $\underline{f}=\left(f_{1}, f_{2}, \ldots, f_{n}\right)^{T}, \underline{y}=\left(y_{1}, y_{2}, \ldots, y_{n}\right)^{T}$, and where the superscript $T$ indicates the transposed. For each $i, \quad 1 \leq i \leq n, y_{i}=y_{i}(t)$, and $f_{i}=f_{i}\left(y_{1}, y_{2}, \ldots, y_{n}, t\right)$. We assume that $f_{1}, f_{2}, \ldots, f_{n}$ are sufficiently smooth such that a twice continuously differentiable first integral

$$
F\left(y_{1}, y_{2}, \ldots, y_{n}, t ; c\right)=0
$$

exists, where $c$ is an arbitrary constant. Furthermore, we assume that there exist continuously differentiable integrating factors $\mu_{1}, \mu_{2}, \ldots, \mu_{n}$ with

$\mu_{i}=\mu_{i}\left(y_{1}, y_{2}, \ldots, y_{n}, t\right), \forall i, i=1,2, \ldots, n$. To obtain the relationships between the first integral and the integrating factors we multiply each $i$-th ODE in (2.1) with the integrating factor $\mu_{i}$, and we then add the so-obtained equations, yielding

$$
\underline{\mu} \cdot \frac{d \underline{y}}{d t}=\underline{\mu} \cdot \underline{f}(\underline{y}, t)
$$

where $\underline{\mu}=\left(\mu_{1}, \mu_{2}, \ldots, \mu_{n}\right)^{T}$. In fact $\underline{\mu}$ can be considered as an integrating vector. This exact $\overline{\mathrm{O}} \mathrm{DE}$ should be the same equation as the equation obtained by differentiating (2.2) with respect to $t$. That is,

$$
\left\{\begin{array}{l}
\mu_{1} \cdot \frac{d y_{1}}{d t}+\mu_{2} \cdot \frac{d y_{2}}{d t}+\ldots+\mu_{n} \cdot \frac{d y_{n}}{d t}-(\underline{\mu} \cdot \underline{f})=0, \text { and } \\
\frac{\partial F}{\partial y_{1}} \cdot \frac{d y_{1}}{d t}+\frac{\partial F}{\partial y_{2}} \cdot \frac{d y_{2}}{d t}+\ldots+\frac{\partial F}{\partial y_{n}} \cdot \frac{d y_{n}}{d t}+\frac{\partial F}{d t}=0,
\end{array}\right.
$$

are equivalent, and we can write

$$
\left\{\begin{array}{l}
\nabla F=\underline{\mu} \\
\frac{\partial F}{\partial t}=-\underline{\mu} \cdot \underline{f},
\end{array}\right.
$$

where $\nabla=\left(\frac{\partial}{\partial y_{1}}, \frac{\partial}{\partial y_{2}}, \ldots, \frac{\partial}{\partial y_{n}}\right)^{T}$. Eliminating $F$ from (2.5) by differentiations, we obtain

$$
\left\{\begin{array}{l}
\frac{\partial \mu_{i}}{\partial y_{j}}=\frac{\partial \mu_{j}}{\partial y_{i}}, \quad 1 \leq i<j \leq n, \\
\frac{\partial \underline{\mu}}{\partial t}=-\nabla(\underline{\mu} \cdot \underline{f}) .
\end{array}\right.
$$

All integrating vectors $\underline{\mu}$ for the system of ODEs (2.1) have to satisfy the system of $\frac{1}{2} n(n+1)$ first order, linear PDEs (2.6). Now we consider the following system of $n$ first order ODEs

$$
\frac{d \underline{y}}{d t}=\underline{f}(\underline{y}, t ; \epsilon),
$$

where $\epsilon$ is a small parameter, and where the function $\underline{f}$ has the form

$$
\underline{f}(\underline{y}, t ; \epsilon)=\underline{f}_{0}(\underline{y}, t)+\epsilon \underline{f}_{1}(\underline{y}, t) .
$$


An integrating vector $\underline{\mu}=\underline{\mu}(\underline{y}, t ; \epsilon)$ for system (2.7) has to satisfy (2.6). Assume that $\underline{\mu}$ can be expanded in a power series in $\epsilon$, that is,

$$
\left.\underline{\mu}(\underline{y}, t ; \epsilon)=\underline{\mu}_{0}(\underline{y}, t)+\epsilon \underline{\mu}_{1} \underline{y}, t\right)+\ldots+\epsilon^{m} \underline{\mu}_{m}(\underline{y}, t)+\ldots
$$

We determine an integrating vector up to $\mathcal{O}\left(\epsilon^{m}\right)$. An approximation $F_{a p p}$ of $F$ in the first integral $F=$ constant can be obtained from:

$$
\left\{\begin{array}{l}
\nabla F_{a p p}=\underline{\mu}_{0}+\epsilon \underline{\mu}_{1}+\ldots+\epsilon^{m} \underline{\mu}_{m}, \\
\frac{\partial F_{a p p}}{\partial t}=-\left[\left(\underline{\mu}_{0}+\epsilon \underline{\mu}_{1}+\ldots+\epsilon^{m} \underline{\mu}_{m}\right) \cdot \underline{f}\right]_{*},
\end{array}\right.
$$

where the * indicates that terms of order $\epsilon^{m+1}$ and higher have been neglected. Then we obtain

$$
F_{a p p}(\underline{y}, t ; \epsilon)=F_{0}(\underline{y}, t)+\epsilon F_{1}(\underline{y}, t)+\ldots+\epsilon^{m} F_{m}(\underline{y}, t) .
$$

It should be observed that an approximation up to $\mathcal{O}\left(\epsilon^{m}\right)$ of an integrating vector $\underline{\mu}$ has been used to obtain an exact ODEs up to $\mathcal{O}\left(\epsilon^{m+1}\right)$, that is,

$$
\begin{aligned}
\frac{d F_{a p p}}{d t} & =\frac{d}{d t}\left(F_{0}+\epsilon F_{1}+\ldots+\epsilon^{m} F_{m}\right) \\
& =\nabla F_{a p p} \cdot \frac{d \underline{y}}{d t}+\frac{\partial F_{a p p}}{\partial t} \\
& =\left[\left(\underline{\mu}_{0}+\epsilon \cdot \underline{\mu}_{1}+\ldots+\epsilon^{m} \underline{\mu}_{m}\right) \cdot \underline{f}\right]_{* *} \\
& =\epsilon^{m+1} R_{m+1}\left(\underline{y}, t, \underline{\mu}_{0}, \ldots, \underline{\mu}_{m} ; \epsilon\right),
\end{aligned}
$$

where the ${ }^{* *}$ indicates that only terms of order $\epsilon^{m+1}$ and higher are included. Let initial values for problem (2.7) be given for $t=0$. Then for $\epsilon \rightarrow 0$ it follows from (2.12) that (when $R_{m+1}$ is bounded):

$$
\begin{gathered}
F_{a p p}(\underline{y}(t), t ; \epsilon)-F_{a p p}(\underline{y}(0), 0 ; \epsilon)=\epsilon^{m+1} \int_{0}^{t} R_{m+1}(\ldots) d t \Rightarrow \\
F_{a p p}(\underline{y}, t ; \epsilon)=\text { constant }+\mathcal{O}\left(\epsilon^{m+1}\right), 0 \leq t \leq T,
\end{gathered}
$$

or

$$
F_{\text {app }}(\underline{y}, t ; \epsilon)=\mathrm{constant}+\mathcal{O}\left(\epsilon^{m}\right), 0 \leq t \leq \frac{L}{\epsilon},
$$

where $T$ and $L$ are $\epsilon$-independent constants.

3. Approximations of First Integrals. In this section we will show how the perturbation method based on integrating factors can be applied to approximate first integrals for a generalized Rayleigh oscillator. In the first part of this section we will consider the linear, perturbed case and in the second part the nonlinear, perturbed case. 
3.1. The case $b=\mathcal{O}(\epsilon)$ and $c=\epsilon^{2}$. Consider the mathematical model which describes the flow-induced vibrations of an oscillator with one degree of freedom in a uniform windfield

$$
\ddot{Z}+Z+\epsilon a Z^{2}=\epsilon^{2}\left(1-\frac{\dot{Z}^{2}}{3}\right) \dot{Z},
$$

where $0<\epsilon<<1$ and $a>0$. To analyze equation (3.1), the equation is first written as a system of first order ODEs. Let $X_{1}=Z, X_{2}=\dot{Z}$, from (3.1) we obtain

$$
\left\{\begin{array}{l}
\dot{X}_{1}=X_{2}, \\
\dot{X}_{2}=-X_{1}-\epsilon a X_{1}^{2}+\epsilon^{2}\left(1-\frac{X_{2}^{2}}{3}\right) X_{2} .
\end{array}\right.
$$

In polar coordinates $r$ and $\theta$ (where $X_{1}=r \cos \theta$ and $X_{2}=r \sin \theta$ ) system (3.2) becomes

$$
\left\{\begin{array}{l}
\frac{d r}{d t}=-\epsilon a r^{2} \cos ^{2} \theta \sin \theta+\epsilon^{2}\left(1-\frac{r^{2} \sin ^{2} \theta}{3}\right) r \sin ^{2} \theta=f_{1}(r, \theta) \\
\frac{d \theta}{d t}=-1-\epsilon \operatorname{ar} \cos ^{3} \theta+\epsilon^{2}\left(1-\frac{r^{2} \sin ^{2} \theta}{3}\right) \cos \theta \sin \theta=f_{2}(r, \theta)
\end{array}\right.
$$

Multiplying the first equation in (3.3) by $\mu_{1}$ and the second one by $\mu_{2}$ respectively, it follows from (2.6) that the integrating factors $\mu_{1}$ and $\mu_{2}$ have to satisfy

$$
\left\{\begin{array}{l}
\frac{\partial \mu_{1}}{\partial \theta}=\frac{\partial \mu_{2}}{\partial r} \\
\frac{\partial \mu_{1}}{\partial t}=-\frac{\partial}{\partial r}\left(\mu_{1} f_{1}+\mu_{2} f_{2}\right) \\
\frac{\partial \mu_{2}}{\partial t}=-\frac{\partial}{\partial \theta}\left(\mu_{1} f_{1}+\mu_{2} f_{2}\right) .
\end{array}\right.
$$

Expanding $\mu_{1}$ and $\mu_{2}$ in power series in $\epsilon$, that is,

$$
\left\{\begin{array}{l}
\mu_{1}(r, \theta, t ; \epsilon)=\mu_{1,0}(r, \theta, t)+\epsilon \mu_{1,1}(r, \theta, t)+\epsilon^{2} \mu_{1,2}(r, \theta, t)+\ldots \\
\mu_{2}(r, \theta, t ; \epsilon)=\mu_{2,0}(r, \theta, t)+\epsilon \mu_{2,1}(r, \theta, t)+\epsilon^{2} \mu_{2,2}(r, \theta, t)+\ldots
\end{array}\right.
$$

substituting $f_{1}, f_{2}$ and the expansions for $\mu_{1}$ and $\mu_{2}$ into (3.4), and by taking together terms of equal powers in $\epsilon$, we finally obtain

$$
\mathcal{O}\left(\epsilon^{0}\right):\left\{\begin{array}{l}
\frac{\partial \mu_{1,0}}{\partial \theta}=\frac{\partial \mu_{2,0}}{\partial r} \\
\frac{\partial \mu_{1,0}}{\partial t}=\frac{\partial \mu_{2,0}}{\partial r} \\
\frac{\partial \mu_{2,0}}{\partial t}=\frac{\partial \mu_{2,0}}{\partial \theta}
\end{array}\right.
$$

$(3.7) \mathcal{O}\left(\epsilon^{1}\right):\left\{\begin{array}{l}\frac{\partial \mu_{1,1}}{\partial \theta}=\frac{\partial \mu_{2,1}}{\partial r}, \\ \frac{\partial \mu_{1,1}}{\partial t}=\frac{\partial}{\partial r}\left(\mu_{1,0} a r^{2} \cos ^{2} \theta \sin \theta+\mu_{2,1}+\mu_{2,0} a r \cos ^{3} \theta\right), \\ \frac{\partial \mu_{2,1}}{\partial t}=\frac{\partial}{\partial \theta}\left(\mu_{1,0} a r^{2} \cos ^{2} \theta \sin \theta+\mu_{2,1}+\mu_{2,0} a r \cos ^{3} \theta\right),\end{array}\right.$ 
and $\mathcal{O}\left(\epsilon^{n}\right)$ with $n \geq 2$ :

$$
\left\{\begin{aligned}
\frac{\partial \mu_{1, n}}{\partial \theta}= & \frac{\partial \mu_{2, n}}{\partial r} \\
\frac{\partial \mu_{1, n}}{\partial t}= & \frac{\partial}{\partial r}\left(\mu_{1, n-1} a r^{2} \cos ^{2} \theta \sin \theta-\mu_{1, n-2}\left(1-\frac{r^{2} \sin ^{2} \theta}{3}\right) r \sin ^{2} \theta\right. \\
& \left.+\mu_{2, n}+\mu_{2, n-1} \operatorname{ar} \cos ^{3} \theta-\mu_{2, n-2}\left(1-\frac{r^{2} \sin ^{2} \theta}{3}\right) \cos ^{3} \theta \sin \theta\right) \\
\frac{\partial \mu_{2, n}}{\partial t}= & \frac{\partial}{\partial \theta}\left(\mu_{1, n-1} a r^{2} \cos ^{2} \theta \sin \theta-\mu_{1, n-2}\left(1-\frac{r^{2} \sin ^{2} \theta}{3}\right) r \sin ^{2} \theta\right. \\
& \left.+\mu_{2, n}+\mu_{2, n-1} \operatorname{ar} \cos ^{3} \theta-\mu_{2, n-2}\left(1-\frac{r^{2} \sin ^{2} \theta}{3}\right) \cos ^{3} \theta \sin \theta\right)
\end{aligned}\right.
$$

The $\mathcal{O}\left(\epsilon^{0}\right)$-problem (3.6) can be solved, yielding $\mu_{1,0}=h_{1,0}(r, \theta+t)$ and $\mu_{2,0}=$ $h_{2,0}(r, \theta+t)$, with $\frac{\partial h_{1,0}}{\partial \theta}=\frac{\partial h_{2,0}}{\partial r}$. The functions $h_{1,0}$ and $h_{20}$ are still arbitrary and will now be chosen as simple as possible. We choose $h_{1,0} \equiv 1$ and $h_{2,0} \equiv 0$, and so

$$
\mu_{1,0}=1, \quad \mu_{2,0}=0 .
$$

The $\mathcal{O}\left(\epsilon^{1}\right)$-problem (3.7) then becomes:

$$
\left\{\begin{array}{l}
\frac{\partial \mu_{1,1}}{\partial \theta}=\frac{\partial \mu_{2,1}}{\partial r}, \\
\frac{\partial \mu_{1,1}}{\partial t}=2 a r \cos ^{2} \theta \sin \theta \frac{\partial \mu_{2,1}}{\partial r}, \\
\frac{\partial \mu_{2,1}}{\partial t}=-2 a r^{2} \cos \theta \sin ^{2} \theta+a r^{2} \cos ^{3} \theta \frac{\partial \mu_{2,1}}{\partial \theta} .
\end{array}\right.
$$

The $\mathcal{O}\left(\epsilon^{1}\right)$-problem $(3.10)$ can also readily be solved, yielding

$$
\left\{\begin{array}{l}
\mu_{1,1}=h_{1,1}(r, \theta+t)+\frac{2}{3} \operatorname{ar} \cos ^{3} \theta, \\
\mu_{2,1}=h_{2,1}(r, \theta+t)-a r^{2} \sin \theta+a r^{2} \sin ^{3} \theta,
\end{array}\right.
$$

with $\frac{\partial h_{1,1}}{\partial \theta}=\frac{\partial h_{2,1}}{\partial r}$. The functions $h_{1,1}$ and $h_{2,1}$ are still arbitrary. We choose these functions as simple as possible: $h_{1,1} \equiv h_{2,1} \equiv 0$. And we obtain

$$
\left\{\begin{array}{l}
\mu_{1,1}=\frac{2}{3} \operatorname{arcos}^{3} \theta \\
\mu_{2,1}=-a r^{2} \sin \theta+a r^{2} \sin ^{3} \theta .
\end{array}\right.
$$

The $\mathcal{O}\left(\epsilon^{2}\right)$-problem (3.8) now becomes

$$
\left\{\begin{aligned}
\frac{\partial \mu_{1,2}}{\partial \theta}= & \frac{\partial \mu_{2,2}}{\partial r} \\
\frac{\partial \mu_{1,2}}{\partial t}= & \frac{\partial}{\partial r}\left(\frac{2}{3} a^{2} r^{3} \cos ^{5} \theta \sin \theta-\left(1-\frac{r^{2} \sin ^{2} \theta}{3}\right) r \sin ^{2} \theta\right. \\
& \left.\left(-a r^{2} \sin ^{2} \theta+a r^{2} \sin ^{3} \theta\right) a r \cos ^{3} \theta+\mu_{2,2}\right), \\
\frac{\partial \mu_{2,2}}{\partial t}= & \frac{\partial}{\partial \theta}\left(\frac{2}{3} a^{2} r^{3} \cos ^{5} \theta \sin \theta-\left(1-\frac{r^{2} \sin ^{2} \theta}{3}\right) r \sin ^{2} \theta\right. \\
& \left.\left(-a r^{2} \sin ^{2} \theta+a r^{2} \sin ^{3} \theta\right) a r \cos ^{3} \theta+\mu_{2,2}\right) .
\end{aligned}\right.
$$


The solution of (3.13) is given by

$$
\left\{\begin{aligned}
\mu_{1,2}= & h_{1,2}(r, \theta+t)+\left(-\frac{r}{2}+\frac{3}{8} r^{2}\right) \theta-\frac{1}{4} \sin 2 \theta-\frac{1}{4} r^{2} \sin 2 \theta \\
& +\frac{5}{64} a^{2} r^{2} \cos 2 \theta+\frac{1}{32} r^{2} \sin 4 \theta+\frac{1}{32} a^{2} r^{2} \cos 4 \theta+\frac{1}{192} a^{2} r^{2} \cos 6 \theta \\
\mu_{2,2}= & h_{2,2}(r, \theta+t)-\frac{r}{2} \cos 2 \theta+\frac{1}{6} r^{3} \cos 2 \theta+\frac{5}{96} a^{2} r^{3} \sin 2 \theta \\
& -\frac{r^{3}}{24} \cos 4 \theta+\frac{1}{24} a^{2} r^{3} \sin 4 \theta+\frac{1}{96} a^{2} r^{3} \sin 6 \theta
\end{aligned}\right.
$$

with $\left(\frac{1}{2}-\frac{3}{8} r^{2}\right)+\frac{\partial h_{1,2}}{\partial \theta}=\frac{\partial h_{2,2}}{\partial r}$. The functions $h_{1,2}$ and $h_{2,2}$ are still arbitrary. We choose these functions as simple as possible

$$
h_{1,2} \equiv 0, \quad h_{2,2} \equiv \frac{1}{2} r-\frac{1}{8} r^{3}
$$

and we obtain

$$
\left\{\begin{aligned}
\mu_{1,2}= & \left(-\frac{r}{2}+\frac{3}{8} r^{2}\right) \theta-\frac{1}{4} \sin 2 \theta-\frac{1}{4} r^{2} \sin 2 \theta+\frac{5}{64} a^{2} r^{2} \cos 2 \theta \\
& +\frac{1}{32} r^{2} \sin 4 \theta+\frac{1}{32} a^{2} r^{2} \cos 4 \theta+\frac{1}{192} a^{2} r^{2} \cos 6 \theta \\
\mu_{2,2}= & \left(\frac{1}{2} r-\frac{1}{8} r^{3}\right)-\frac{r}{2} \cos 2 \theta+\frac{1}{6} r^{3} \cos 2 \theta+\frac{5}{96} a^{2} r^{3} \sin 2 \theta \\
& -\frac{r^{3}}{24} \cos 4 \theta+\frac{1}{24} a^{2} r^{3} \sin 4 \theta+\frac{1}{96} a^{2} r^{3} \sin 6 \theta
\end{aligned}\right.
$$

The $\mathcal{O}\left(\epsilon^{n}\right)$-problems (3.8) with $n>2$ can be solved in a similar way. An approximation $F_{1}$ of a first integral $F=$ constant can be obtained from (3.9), (3.12), (3.16) and (2.10), yielding

$$
\begin{aligned}
F_{1}(r, \theta, t ; \epsilon)= & r+\epsilon a r^{2} \cos ^{3} \theta+\epsilon^{2}\left\{\left(\frac{1}{2} r-\frac{1}{8} r^{3}\right) \theta-\frac{1}{4} r \sin 2 \theta\right. \\
& +\frac{1}{12} r^{3} \sin 2 \theta-\frac{5}{192} a^{2} r^{3} \cos 2 \theta-\frac{1}{96} r^{3} \sin 4 \theta \\
& \left.-\frac{1}{96} a^{2} r^{3} \cos 4 \theta-\frac{1}{576} a^{2} r^{3} \cos 6 \theta\right\} .
\end{aligned}
$$

How well $F_{1}$ approximates $F$ in a first integral $F=$ constant follows from (2.12)(2.14). In this case we have

$$
\begin{aligned}
\frac{d F_{1}}{d t} & =\left[\left(\underline{\mu}_{0}+\epsilon \underline{\mu}_{1}+\ldots+\epsilon^{m} \underline{\mu}_{m}\right) \cdot \underline{f}\right]-\left[\left(\underline{\mu}_{0}+\epsilon \underline{\mu}_{1}+\ldots+\epsilon^{m} \underline{\mu}_{m}\right) \cdot \underline{f}\right]_{*} \\
& =\left[f_{1}+\epsilon \mu_{1,1} f_{1}+\epsilon^{2} \mu_{1,2} f_{1}+\epsilon \mu_{2,1} f_{2}+\epsilon^{2} \mu_{2,2} f_{2}\right]_{* *} \\
& =\epsilon^{3} R_{3}(r, \theta),
\end{aligned}
$$

where $f_{1}, f_{2}, \mu_{1,1}, \mu_{2,1}, \mu_{1,2}, \mu_{2,2}$ are given by $(3.3),(3.12)$ and (3.16). From the existence and uniqueness theorems for ODEs we know that an initial-value problem for system (3.2) is well-posed on a time-scale of order $\frac{1}{\epsilon}$. This implies that also an initialvalue problem for system (3.3) is well-posed on this time-scale. From (3.3) it then follows on this time-scale that if $r(0)$ is bounded then $r(t)$ is bounded and $\theta(t)$ is 
bounded by a constant plus $t$. Since $\left|R_{3}\right| \leq c_{0}+c_{1} t$ on a time scale of order $\frac{1}{\epsilon}$, where $c_{0}, c_{1}$ are constants, it follows from (3.18) that

$$
F_{1}(r(t), \theta(t), t ; \epsilon)=\text { constant }+\epsilon^{3} \int_{0}^{t} R_{3}(r(s), \theta(s), s ; \epsilon) d s
$$

and so,

$$
\begin{aligned}
& F_{1}(r(t), \theta(t), t ; \epsilon)=\text { constant }+\mathcal{O}\left(\epsilon^{3}\right), 0 \leq t \leq T_{0}<\infty, \\
& F_{1}(r(t), \theta(t), t ; \epsilon)=\text { constant }+\mathcal{O}(\epsilon), 0 \leq t \leq \frac{L}{\epsilon},
\end{aligned}
$$

where $T_{0}$ and $L$ are $\epsilon$-independent constants. In a similar way we can construct a second (functionally independent) approximation $F_{2}$ of a first integral by putting

$$
\mu_{1,0}=0, \quad \mu_{2,0}=1
$$

in (3.6) instead of (3.9),

$$
\left\{\begin{array}{l}
\mu_{1,1}=-a \sin \theta+\frac{a}{3} \sin ^{3} \theta \\
\mu_{2,1}=-\operatorname{arcos} \cos ^{3} \theta
\end{array}\right.
$$

in (3.7) instead of (3.12), and so on. After some elementary calculations we then find

$$
\begin{aligned}
F_{2}(r, \theta, t ; \epsilon)= & \theta+t+\epsilon\left(-a r \sin \theta+\frac{a}{3} r \sin ^{3} \theta\right)+\epsilon^{2}\left\{\frac{5}{12} a^{2} r^{2} \theta\right. \\
& +\frac{1}{24} r^{2} \cos 2 \theta-\frac{1}{4} \cos 2 \theta+\frac{23}{96} a^{2} r^{2} \sin 2 \theta-\frac{1}{96} r^{2} \cos 4 \theta \\
& \left.+\frac{1}{48} a^{2} r^{2} \sin 4 \theta+\frac{1}{288} a^{2} r^{2} \sin 6 \theta\right\}
\end{aligned}
$$

3.2. The case $b=\mathcal{O}(1)$ and $c=\epsilon$. We consider in this subsection

$$
\ddot{Z}+Z+b Z^{2}=\epsilon\left(1-\frac{\dot{Z}^{2}}{3}\right) \dot{Z},
$$

where $b>0$, and $0<\epsilon<<1$. Let $Z=X_{1}, \dot{Z}=X_{2}$, from (3.24) we obtain

$$
\left\{\begin{array}{lll}
\dot{X}_{1}=X_{2} & = & g_{1}\left(X_{1}, X_{2}\right), \\
\dot{X}_{2}=-X_{1}-b X_{1}^{2}+\epsilon\left(1-\frac{X_{2}^{2}}{3}\right) X_{2} & =g_{2}\left(X_{1}, X_{2}\right) .
\end{array}\right.
$$

Multiplying the first equation in (3.25) by the integrating factor $\mu_{1}$ and the second one by $\mu_{2}$, it follows from (2.6) that the integrating factors $\mu_{1}$ and $\mu_{2}$ have to satisfy

$$
\left\{\begin{aligned}
\frac{\partial \mu_{1}}{\partial X_{2}} & =\frac{\partial \mu_{2}}{\partial X_{1}} \\
\frac{\partial \mu_{1}}{\partial t} & =-\frac{\partial}{\partial X_{1}}\left(\mu_{1} g_{1}+\mu_{2} g_{2}\right) \\
\frac{\partial \mu_{2}}{\partial t} & =-\frac{\partial}{\partial X_{2}}\left(\mu_{1} g_{1}+\mu_{2} g_{2}\right)
\end{aligned}\right.
$$


For a time-independent first integral, the integrating factors $\mu_{1}$ and $\mu_{2}$ have to satisfy

$$
\left\{\begin{array}{l}
\mu_{1} g_{1}+\mu_{2} g_{2}=0 \\
\frac{\partial \mu_{1}}{\partial X_{2}}=\frac{\partial \mu_{2}}{\partial X_{1}}
\end{array}\right.
$$

where $\mu_{1}=\mu_{1}\left(X_{1}, X_{2} ; \epsilon\right)$ and $\mu_{2}=\mu_{2}\left(X_{1}, X_{2} ; \epsilon\right)$. We assume that the integrating factors $\mu_{1}$ and $\mu_{2}$ can be expanded in power series in $\epsilon$, that is,

$$
\left\{\begin{array}{l}
\mu_{1}=\mu_{1,0}\left(X_{1}, X_{2}\right)+\epsilon \mu_{1,1}\left(X_{1}, X_{2}\right)+\ldots, \\
\mu_{2}=\mu_{2,0}\left(X_{1}, X_{2}\right)+\epsilon \mu_{2,1}\left(X_{1}, X_{2}\right)+\ldots
\end{array}\right.
$$

The expansions (3.28) are substituted into (3.27) and terms of equal power in $\epsilon$ are taken together, yielding

$$
\mathcal{O}\left(\epsilon^{0}\right):\left\{\begin{array}{l}
\frac{\partial \mu_{1,0}}{\partial X_{2}}=\frac{\partial \mu_{2,0}}{\partial X_{1}}, \\
\mu_{1,0} X_{2}+\mu_{2,0}\left(-X_{1}-b X_{1}^{2}\right)=0,
\end{array}\right.
$$

$$
\mathcal{O}\left(\epsilon^{1}\right):\left\{\begin{array}{l}
\frac{\partial \mu_{1,1}}{\partial X_{2}}=\frac{\partial \mu_{2,1}}{\partial X_{1}}, \\
\mu_{1,1} X_{2}+\mu_{2,1}\left(-X_{1}-b X_{1}^{2}\right)+\mu_{2,0}\left(1-\frac{X_{2}^{2}}{3}\right) X_{2}=0,
\end{array}\right.
$$

and the $\mathcal{O}\left(\epsilon^{n}\right)$-terms with $n \geq 2$ are

$$
\left\{\begin{array}{l}
\frac{\partial \mu_{1, n}}{\partial X_{2}}=\frac{\partial \mu_{2, n}}{\partial X_{1}} \\
\mu_{1, n} X_{2}+\mu_{2, n-1}\left(1-\frac{X_{2}^{2}}{3}\right) X_{2}+\mu_{2, n}\left(-X_{1}-b X_{1}^{2}\right)=0 .
\end{array}\right.
$$

From (3.29) it follows that

$$
\frac{\partial \mu_{2,0}}{\partial X_{1}}-\frac{1}{X_{2}}\left(X_{1}+b X_{1}^{2}\right) \frac{\partial \mu_{2,0}}{\partial X_{2}}+\frac{1}{X_{2}^{2}}\left(X_{1}+b X_{1}^{2}\right) \mu_{2,0}=0 .
$$

Using the method of characteristics we easily find the general solution of the PDE (3.32)

$$
\mu_{2,0}=f\left(\frac{1}{2} X_{2}^{2}+\frac{1}{2} X_{1}^{2}+\frac{1}{3} b X_{1}^{3}\right) X_{2},
$$

where $f$ is an arbitrary function. From (3.29) $\mu_{1,0}$ then also easily follows. Now take $\mu_{2,0}$ and $\mu_{1,0}$ as simple as possible, for instance

$$
\left\{\begin{array}{l}
\mu_{2,0}=X_{2}, \\
\mu_{1,0}=X_{1}+b X_{1}^{2} .
\end{array}\right.
$$

Using (3.34) the $\mathcal{O}\left(\epsilon^{1}\right)$-problem (3.30) can then be rewritten as

$$
\frac{\partial \mu_{2,1}}{\partial X_{1}}-\frac{1}{X_{2}}\left(X_{1}+b X_{1}^{2}\right) \frac{\partial \mu_{2,1}}{\partial X_{2}}+\frac{1}{X_{2}^{2}}\left(X_{1}+b X_{1}^{2}\right) \mu_{2,1}+\left(1-X_{2}^{2}\right)=0
$$


The corresponding characteristic ODEs of this PDE (3.35) are

$$
\left\{\begin{array}{l}
\frac{d X_{1}}{d s}=1 \\
\frac{d X_{2}}{d s}=-\frac{1}{X_{2}}\left(X_{1}+b X_{1}^{2}\right) \\
\frac{d \mu_{2,1}}{d s}=-\frac{1}{X_{2}^{2}}\left(X_{1}+b X_{1}^{2}\right) \mu_{2,1}-\left(1-X_{2}^{2}\right) .
\end{array}\right.
$$

It follows from (3.36) that

$$
\begin{gathered}
\frac{d X_{1}}{d X_{2}}=\frac{-X_{2}}{X_{1}+b X_{1}^{2}} \Rightarrow \frac{1}{2} X_{1}^{2}+\frac{1}{3} b X_{1}^{3}+\frac{1}{2} X_{2}^{2}=k_{1}, \\
\frac{d \mu_{2,1}}{d X_{1}}=-\frac{1}{X_{2}^{2}}\left(X_{1}+b X_{1}^{2}\right) \mu_{2,1}-\left(1-X_{2}^{2}\right),
\end{gathered}
$$

where $k_{1}$ is a constant of integration. Using (3.37) it follows from (3.38) that the solution of the homogeneous equation (3.38) is

$$
\mu_{2,1_{h}}=C \sqrt{\left(k_{1}-\frac{1}{2} X_{1}^{2}-\frac{1}{3} b X_{1}^{3}\right)},
$$

where $C$ is constant. The general solution of the inhomogeneous equation (3.38) then easily follows by applying the method of variation of parameter, yielding

$$
\begin{aligned}
\mu_{2,1}= & \left\{\int_{0}^{X_{1}} \frac{\left(-1+2 k_{1}-r^{2}-\frac{2}{3} b r^{3}\right)}{\sqrt{\left(k_{1}-\frac{1}{2} r^{2}-\frac{2}{3} b r^{3}\right)}} d r+k_{2}\right\} \\
& \sqrt{\left(k_{1}-\frac{1}{2} X_{1}^{2}-\frac{1}{3} b X_{1}^{3}\right)}
\end{aligned}
$$

where $k_{2}$ is a constant of integration. From (3.30) we obtain

$$
\begin{aligned}
\mu_{1,1}= & \frac{1}{X_{2}}\left(X_{1}+b X_{1}^{2}\right) \mu_{2,1}-\left(X_{2}-\frac{X_{2}^{3}}{3}\right) \\
= & \left\{\int_{0}^{X_{1}} \frac{\left(-1+2 k_{1}-r^{2}-\frac{2}{3} b r^{3}\right)}{\sqrt{\left(k_{1}-\frac{1}{2} r^{2}-\frac{2}{3} b r^{3}\right)}} d r+k_{2}\right\} \\
& \frac{1}{2} \sqrt{2}\left(X_{1}+b X_{1}^{2}\right)-\left(X_{2}-\frac{X_{2}^{3}}{3}\right) .
\end{aligned}
$$

In (3.40) and (3.41) $k_{2}$ can be considered as an arbitrary function of $k_{1}$ and so $k_{2}=$ $g\left(\frac{1}{2} X_{1}^{2}+\frac{1}{3} b X_{1}^{3}+\frac{1}{2} X_{2}^{2}\right)$, where $g$ is an arbitrary function. We will take $g \equiv 0$ since we are interested in approximations of first integrals that are as simple as possible. An approximation $F_{1,1}$ of $F$ in a time-independent first integral $F\left(X_{1}, X_{2} ; \epsilon\right)=$ constant can be obtained from (3.34), (3.40), (3.41) and (2.5), and

$$
\left\{\begin{array}{l}
\nabla F_{1,1}=\underline{\mu}_{0}+\epsilon \underline{\mu}_{1}, \\
\frac{\partial F_{1,1}}{\partial t}=0,
\end{array}\right.
$$


yielding

$$
\begin{aligned}
& F_{1,1}\left(X_{1}, X_{2} ; \epsilon\right)= \\
& \frac{1}{2} X_{1}^{2}+\frac{1}{3} b X_{1}^{3}+\frac{1}{2} X_{2}^{2}+\epsilon\left[\frac{1}{4} \sqrt{2}\left\{\int_{0}^{X_{1}} \frac{\left(-1+2 k_{1}-r^{2}-\frac{2}{3} b r^{3}\right)}{\sqrt{\left(k_{1}-\frac{1}{2} r^{2}-\frac{2}{3} b r^{3}\right)}} d r\right\}\right. \\
& +\frac{1}{2} \sqrt{2}\left(-k_{1}+\frac{1}{2} X_{1}^{2}+\frac{1}{3} b X_{1}^{3}\right)\left\{\int_{0}^{X_{1}} \frac{\left(-1+2 k_{1}-r^{2}-\frac{2}{3} b r^{3}\right)}{\sqrt{\left(k_{1}-\frac{1}{2} r^{2}-\frac{2}{3} b r^{3}\right)}} d r\right\} \\
& \left.+\int_{0}^{X_{1}} \sqrt{2\left(k_{1}-\frac{1}{2} r^{2}-\frac{1}{3} b r^{3}\right)}\left(\frac{1}{3}\left(2 k_{1}-r^{2}-\frac{2}{3} b r^{3}\right)-1\right) d r\right] .
\end{aligned}
$$

How well $F_{1,1}$ approximates $F$ can be shown by differentiating $F_{1,1}$ with respect to $t$, that is,

$$
\frac{d F_{1,1}}{d t}=\epsilon^{2}\left(\frac{1}{2} \sqrt{2} X_{2}\left(X_{2}-\frac{X_{2}^{3}}{3}\right)\left\{\int_{0}^{X_{1}} \frac{-1+2 k_{1}-r^{2}-\frac{2}{3} b r^{3}}{\sqrt{k_{1}-\frac{1}{2} r^{2}-\frac{1}{3} b r^{3}}} d r\right\}\right)
$$

From the existence and uniqueness theorems for ODEs we know that initial value problems for system (3.25) are well-posed on $0 \leq t \leq T_{0}<\infty$. This implies that system (3.44) is well-posed on $0 \leq t \leq T_{0}$. So, we have

$$
F_{1,1}\left(X_{1}, X_{2}\right)=\text { constant }+\mathcal{O}\left(\epsilon^{2}\right), \quad 0 \leq t \leq T_{0},
$$

where $T_{0}$ is an $\epsilon$-independent constant.

4. Approximations for time-periodic solutions. In section 3 we constructed asymptotic approximations of first integrals. In this section we will show how the existence, the stability, and the approximations of non-trivial, time-periodic solutions can be determined from these asymptotic approximations of the first integrals.

4.1. Time-periodic solutions when $b=\mathcal{O}(\epsilon)$ and $c=\epsilon^{2}$. Using these approximations it is possible to study the existence and stability of time-periodic solutions. Let $T<\infty$ be the period of a periodic solution and let $c_{1}$ be a constant in the first integral $F(r, \theta, t ; \epsilon)=$ constant for which a periodic solution exists. Consider $F=c_{1}$ for $t=n T$ and $t=(n-1) T$ with $n \in N^{+}$, then

$$
\begin{cases}F(r(n T), \theta(T), n T ; \epsilon) & =c_{1}, \\ F(r((n-1) T), \theta((n-1) T),(n-1) T ; \epsilon) & =c_{1} .\end{cases}
$$

For the autonomous equation (3.3) we may assume without loss of generality that $\theta(0)=0$. From (3.3) it follows that

$$
\left\{\begin{array}{l}
r(n T)=r((n-1) T)+\mathcal{O}(\epsilon) \\
\theta(n T)=\theta((n-1) T)-T+\mathcal{O}(\epsilon) .
\end{array}\right.
$$


Approximating $F$ by $F_{1}$ (given by (3.17)), eliminating $c_{1}$ from (4.1) and using (4.2), we obtain

$$
\begin{aligned}
r(n T)= & r((n-1) T)+\epsilon^{2} 2 \pi\left(\frac{1}{2} r((n-1) T)\right. \\
& \left.-\frac{1}{8} r^{3}((n-1) T)\right)+\mathcal{O}\left(\epsilon^{3} t\right) .
\end{aligned}
$$

on a time scale of order $\frac{1}{\epsilon}$. In fact (4.3) defines a map $Q: r \rightarrow Q(r) \Leftrightarrow r_{n}=Q\left(r_{n-1}\right)$ with $r_{n}=r(n T)$. We define a new map $P$ by neglecting the term of $O\left(\epsilon^{3} t\right)$ in (4.3). That is, $P: \tilde{r} \rightarrow P(\tilde{r}) \Leftrightarrow \tilde{r}_{n}=P\left(\tilde{r}_{n-1}\right)$ with $\tilde{r}_{n}=\tilde{r}(n T)$. It will be shown that for $r>0$ :

(i) If $\left|r_{0}-\tilde{r}_{0}\right|=\mathcal{O}(\epsilon)$ for $\epsilon \downarrow 0$ then $\left|r_{n}-\tilde{r}_{n}\right|=\mathcal{O}(\epsilon)$ for $n=\mathcal{O}\left(\frac{1}{\epsilon}\right)$, that is, for $n \sim \frac{1}{\epsilon}$ and $\epsilon \downarrow 0, r_{n}$ and $\tilde{r}_{n}$ remain " $\epsilon$-close".

(ii) The map $P$ has a unique, hyperbolic fixed point $\tilde{r}=2$, which is asymptotically stable.

(iii) There exists an $\epsilon_{0}>0$ such that for all $0<\epsilon \leq \epsilon_{0}$ the map $Q$ has a unique hyperbolic fixed point $r=2+\mathcal{O}(\epsilon)$ with the same stability property as the fixed point $\tilde{r}=2$ of the map $P$.

Proof of (i): From $\left|r_{0}-\tilde{r}_{0}\right|=\mathcal{O}(\epsilon)$ for $\epsilon \downarrow 0$ it follows that there exists a positive constant $M_{0}$ such that $\left|r_{0}-\tilde{r}_{0}\right|=M_{0} \epsilon$. We have

$$
\begin{aligned}
\left|r_{n}-\tilde{r}_{n}\right| & =\left|P\left(r_{n-1}\right)-P\left(\tilde{r}_{n-1}\right)+\mathcal{O}\left(\epsilon^{3} n\right)\right| \\
& \leq\left|P\left(r_{n-1}\right)-P\left(\tilde{r}_{n-1}\right)\right|+M_{1} \epsilon^{3} n \\
& \leq L\left|r_{n-1}-\tilde{r}_{n-1}\right|+M_{1} \epsilon^{3} n,
\end{aligned}
$$

where $M_{1}$ and $\mathrm{L}$ are positive constants, with $L=1+\epsilon^{2} M_{2}$ and $M_{2}$ a positive constant. So we have

$$
\begin{aligned}
\left|r_{n}-\tilde{r}_{n}\right| & \leq\left(1+\epsilon^{2} M_{2}\right)\left|r_{n-1}-\tilde{r}_{n-1}\right|+M_{1} \epsilon^{3} n \leq \ldots \\
& \leq \epsilon\left(M_{0}+\epsilon^{2} n^{2} M_{1}\right) e^{\epsilon^{2} n M_{2}},
\end{aligned}
$$

and so for $n=\mathcal{O}\left(\frac{1}{\epsilon}\right)$ we conclude that $\left|r_{n}-\tilde{r}_{n}\right|=\mathcal{O}(\epsilon)$.

Proof of (ii): The fixed points of the map $P$ follow from $\tilde{r}_{n}=P\left(\tilde{r}_{n-1}\right)$ for $n \rightarrow \infty$ or equivalent from $\tilde{r}=\tilde{r}+\epsilon^{2} 2 \pi\left(\frac{1}{2} \tilde{r}-\frac{1}{8} \tilde{r}^{3}\right) \Leftrightarrow \frac{1}{2} \tilde{r}\left(1-\frac{1}{4} \tilde{r}^{2}\right)=0$. For $\tilde{r}>0$ we have a unique fixed point $\tilde{r}=2$. The fixed point of the map $P$ is hyperbolic if the linearized map around this fixed point has no eigenvalues of unit modulus. Let $D P$ be this linearized map, then $D P=1-\epsilon^{2} 2 \pi$. Since $0<\epsilon<<1$, we have $|\lambda|<1$, and so the fixed point is hyperbolic and stable.

Proof of (iii): For the proof of (iii) we refer to [10] for a similar proof.

So far we conclude that there exists a unique, asymptotically stable, nontrivial, $T$ periodic solution for system (3.1). We can approximate the form and the period $T$ of the limit cycle of system (3.1) from $F(r, \theta, t ; \epsilon)=c_{1}$, where $F$ is approximated by $F_{1}$. For the periodic solution we have $r(0)=r(T)$ and $\theta(T)=\theta(0)-2 \pi$. Without loss of generality we assume that $\theta(0)=0$, and since the system (3.1) is autonomous we know that a time-independent first integral exists. So we have to solve the following 
system of two non-linear equations

$$
\left\{\begin{array}{l}
F(r(0), 0 ; \epsilon)=c_{1}, \\
F(r(T),-2 \pi ; \epsilon)=c_{1},
\end{array}\right.
$$

where $r(0)=r(T), T$ and $c_{1}$ are three unknown constants. Now it should be observed that $F_{1}$ given by (3.17) approximates this time-independent first integral up to $\mathcal{O}\left(\epsilon^{3}\right)$ for $0 \leq t \leq T$. Without loss of generality it can be assumed that the unknown constants $r(0)=r(T)$ and $c_{1}$ can be approximated by $r_{0}+\epsilon r_{1}+\epsilon^{2} r_{2}+\ldots$ and $c_{10}+\epsilon c_{11}+\epsilon^{2} c_{12}+\ldots$ respectively. By substituting these approximations for $r(0)=$ $r(T)$ and $c_{1}$ into (4.6), and by collecting terms of equal powers in $\epsilon$, we obtain the constants $r_{0}, r_{1}, \ldots, c_{10}, c_{11}, \ldots$ after some elementary calculations, yielding

$$
\left\{\begin{array}{l}
r(0)=r(T)=2+\epsilon\left(-\frac{49}{3}\right)+\epsilon^{2}\left(\frac{28}{9} a^{2}\right)+\mathcal{O}\left(\epsilon^{3}\right), \\
c_{1}=2+\epsilon^{2}\left(\frac{37}{36} a^{2}\right)+\mathcal{O}\left(\epsilon^{3}\right) .
\end{array}\right.
$$

From $F(r, \theta, t ; \epsilon)=c_{1}$, where $F$ is approximated by the time-independent function $F_{1}$, given by $(3.17)$, the radius $r$ of the limit cycle can be approximated as function of $\theta$, yielding

$$
\begin{aligned}
r(\theta)= & 2+\epsilon\left\{-\frac{4}{3} a \cos ^{3} \theta\right\}+\epsilon^{2}\left\{\frac{37}{36} a^{2}+\frac{16}{9} a \cos ^{3} \theta\right. \\
& -\frac{1}{2} \sin 2 \theta-\frac{2}{3} \sin 2 \theta+\frac{5}{32} a^{2} \cos 2 \theta+\frac{1}{12} \sin 4 \theta \\
& \left.+\frac{1}{12} a^{2} \cos 4 \theta+\frac{1}{72} a^{2} \cos 6 \theta\right\}+\mathcal{O}\left(\epsilon^{3}\right)
\end{aligned}
$$

By substituting (4.8) into (3.3), and by expanding $\theta(t)$ in $\theta_{0}(t)+\epsilon \theta_{1}(t)+\epsilon^{2} \theta_{2}(t)+\ldots$ and then by expanding the right hand side of (3.3) in $\epsilon$, the functions $\theta_{0}(t), \theta_{1}(t), \ldots$ can be calculated, yielding

$$
\begin{aligned}
\theta(t)= & -t+\epsilon\left\{-\frac{3}{2} a \sin t-\frac{1}{6} \sin 3 t\right\}+\epsilon^{2}\left\{-\frac{7}{32}+\frac{5}{4} a^{2} t\right. \\
& +\frac{3}{8} a^{2} \sin 2 t-\frac{1}{4} a^{2} \sin 4 t-\frac{1}{72} a^{2} \sin 6 t \\
& \left.+\frac{5}{24} \cos 2 t+\frac{1}{96} \cos 4 t\right\}+\mathcal{O}\left(\epsilon^{3}\right) .
\end{aligned}
$$

The period $T$ of the limit cycle can be approximated from $\theta(T)=-2 \pi$. Now let $T=T_{0}+\epsilon T_{1}+\epsilon^{2} T_{2}+\ldots$ and substituting $\theta(T)=-2 \pi$ into (4.9), we obtain

$$
T=2 \pi+\frac{5}{4} a^{2}(2 \pi) \epsilon^{2}+\mathcal{O}\left(\epsilon^{3}\right)
$$

We also can approximate the periodic solution of (3.1), that is, $Z(t)=r(t) \cos (\theta(t))$, where $r(t)$ and $\theta(t)$ are approximated by (4.8) and (4.9).

4.2. Time-periodic solutions when $b=\mathcal{O}(1)$ and $c=\epsilon$. Let $T<\infty$ be the period of a periodic solution and let $c_{1}$ be the constant in the first integral $F=$ 
constant for which a periodic solution exists. Consider $F=c_{1}$ for $t=0$ and $t=T$. Approximating $F$ by $F_{1,1}$ (given by 3.43 ) and eliminating $c_{1}$ by subtraction, we obtain

$$
\epsilon\left\{\int_{X_{1}(0)}^{X_{1}(T)} \sqrt{2\left(k_{1}-\frac{1}{2} r^{2}-\frac{1}{3} b r^{3}\right)}\left(\frac{1}{3}\left(2 k_{1}-r^{2}-\frac{2}{3} b r^{3}\right)-1\right) d r\right\}=\mathcal{O}\left(\epsilon^{2}\right)
$$

$\Leftrightarrow \epsilon I\left(k_{1} ; b\right)+\mathcal{O}\left(\epsilon^{2}\right)=0$, where

$$
I\left(k_{1} ; b\right)=2 \int_{A}^{B} \sqrt{2\left(k_{1}-\frac{1}{2} r^{2}-\frac{1}{3} b r^{3}\right)}\left(\frac{1}{3}\left(2 k_{1}-r^{2}-\frac{2}{3} b r^{3}\right)-1\right) d r
$$

with $A=X_{1}(0)$ and $B=X_{1}\left(\frac{1}{2} T\right)$. To have a periodic solution for (3.24) we have to find a constant $k_{1}$ such that $I\left(k_{1} ; b\right)$ is equal to zero. To find this constant $k_{1}$ we rewrite $I\left(k_{1} ; b\right)$ in

$$
I\left(k_{1} ; b\right)=-I_{1}\left(k_{1} ; b\right)\left(1-\frac{I_{2}\left(k_{1} ; b\right)}{I_{1}\left(k_{1} ; b\right)}\right)
$$

where

$$
\left\{\begin{array}{l}
I_{1}\left(k_{1} ; b\right)=2 \int_{A}^{B}\left(2 k_{1}-r^{2}-\frac{2}{3} b r^{3}\right)^{\frac{1}{2}} d r>0, \\
I_{2}\left(k_{1} ; b\right)=\frac{2}{3} \int_{A}^{B}\left(2 k_{1}-r^{2}-\frac{2}{3} b r^{3}\right)^{\frac{3}{2}} d r>0 .
\end{array}\right.
$$

Now it should be observed that the unperturbed equation (3.24) with $\epsilon=0$ has two equilibrium points: a center point in $(Z, \dot{Z})=(0,0)$ and a saddle point in $(Z, \dot{Z})=$ $\left(-\frac{1}{b}, 0\right)$. The center point "trajectory" and the saddle point loop are represented by $k_{1}=0$ and $k_{1}=\frac{1}{6 b^{2}}$ respectively. It should be observed that if $k_{1} \downarrow 0$ then $A \uparrow 0$ and $B \downarrow 0$, and

$$
\lim _{k_{1} \rightarrow 0} \frac{I_{2}\left(k_{1} ; b\right)}{I_{1}\left(k_{1} ; b\right)}=\lim _{k_{1} \rightarrow 0} \frac{\frac{2}{3} \int_{A}^{B}\left(2 k_{1}-r^{2}-\frac{2}{3} b r^{3}\right)^{\frac{3}{2}} d r}{2 \int_{A}^{B}\left(2 k_{1}-r^{2}-\frac{2}{3} b r^{3}\right)^{\frac{1}{2}} d r}=0 .
$$

And if $k_{1} \uparrow \frac{1}{6 b^{2}}$ then $A \downarrow-\frac{1}{b}$ and $B \uparrow \frac{1}{2 b}$, and

$$
\begin{aligned}
\lim _{k_{1} \rightarrow \frac{1}{6 b^{2}}} \frac{I_{2}\left(k_{1} ; b\right)}{I_{1}\left(k_{1} ; b\right)}= & \lim _{k_{1} \rightarrow \frac{1}{6 b^{2}}} \frac{\frac{1}{3} \int_{A}^{B}\left(2 k_{1}-r^{2}-\frac{2}{3} b r^{3}\right)^{\frac{3}{2}} d r}{\int_{A}^{B}\left(2 k_{1}-r^{2}-\frac{2}{3} b r^{3}\right)^{\frac{1}{2}} d r} \\
& =\frac{\frac{1}{3} \int_{-\frac{1}{b}}^{\frac{1}{2 b}}\left(r+\frac{1}{b}\right)^{3}\left(-\frac{2}{3} b r+\frac{1}{3}\right)^{\frac{3}{2}} d r}{\int_{-\frac{1}{b}}^{\frac{1}{2 b}}\left(r+\frac{1}{b}\right)\left(-\frac{2}{3} b r+\frac{1}{3}\right)^{\frac{1}{2}} d r}=\frac{6}{77 b^{2}} .
\end{aligned}
$$

Let $Q\left(k_{1} ; b\right)=\frac{I_{2}\left(k_{1} ; b\right)}{I_{1}\left(k_{1} ; b\right)}$. Then it can be shown analytically that

$$
\frac{d Q}{d k_{1}}(0 ; b)=\frac{1}{2}>0
$$

and

$$
\frac{d Q}{d k_{1}}\left(\frac{1}{6 b^{2}} ; b\right)=-\infty
$$


For several values of $b$ (see Figure 4.1 ) we have calculated $Q\left(k_{1} ; b\right)$ numerically. The integrals $I_{1}\left(k_{1} ; b\right)$ and $I_{2}\left(k_{1} ; b\right)$ have been determined by using an adaptive recursive Simpson rule and the boundary points $(A$ and $B$ ) have been determined by using a Newton rule. The phase plane portraits are given for several values of $b$ in Figure 4.2.

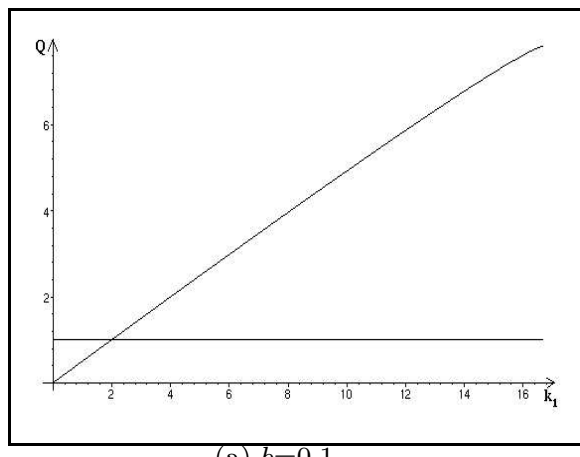

(a) $b=0.1$

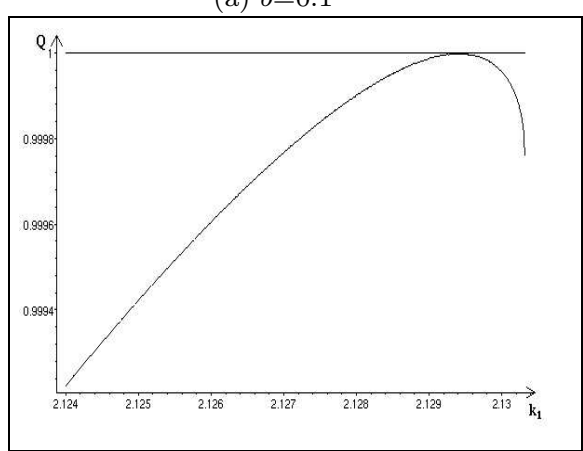

(c) $b=0.2797061632$
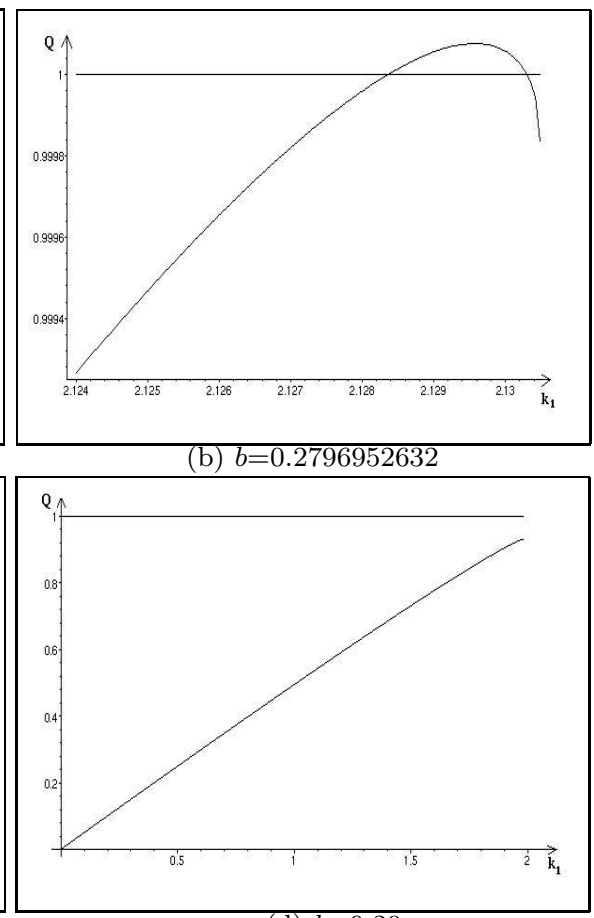

(d) $b=0.29$

FIG. 4.1. Plot of $Q\left(k_{1} ; b\right)$ as function of $k_{1}$ for several values of $b$.

Using numerical calculations (see also Figure 4.1 ) we are able to find $k_{1}$-values such that $I\left(k_{1} ; b\right)=0$ or $Q\left(k_{1} ; b\right)=1$. We obtain the following results:

(i) for $b<\sqrt{\frac{6}{77}}+\mathcal{O}\left(\epsilon^{2}\right)$, there is only one nontrivial value of $k_{1}$ such that $I\left(k_{1} ; b\right)=0$. This implies that there exists only one periodic solution.

(ii) for $\sqrt{\frac{6}{77}}+\mathcal{O}\left(\epsilon^{2}\right) \leq b \leq \sqrt{\frac{6}{77}}+\delta$, there are two nontrivial values for $k_{1}$ such that $I\left(k_{1} ; b\right)=0$. This implies that there are two periodic solutions. It should be remarked that $\delta$ is very small; $\delta$ is approximately equal to $1.09 \times 10^{-5}$.

(iii) for $b>\sqrt{\frac{6}{77}}+\delta$, there are no values of $k_{1}$ such that $I\left(k_{1} ; b\right)=0$, and so there are no periodic solutions.

To determine the stability of the time-periodic solutions we consider the approximation $F_{1,1}$ of a first integral

$$
\begin{aligned}
F_{1,1}= & \frac{1}{2} X_{1}^{2}+\frac{1}{3} b X_{1}^{3}+\frac{1}{2} X_{2}^{2}+\epsilon\left\{\int_{0}^{X_{1}} \sqrt{\left(2 k_{1}-r^{2}-\frac{2}{3} b r^{3}\right)}\right. \\
& \left.\left(\frac{1}{3}\left(2 k_{1}-r^{2}-\frac{2}{3} b r^{3}\right)-1\right)\right\} d r
\end{aligned}
$$



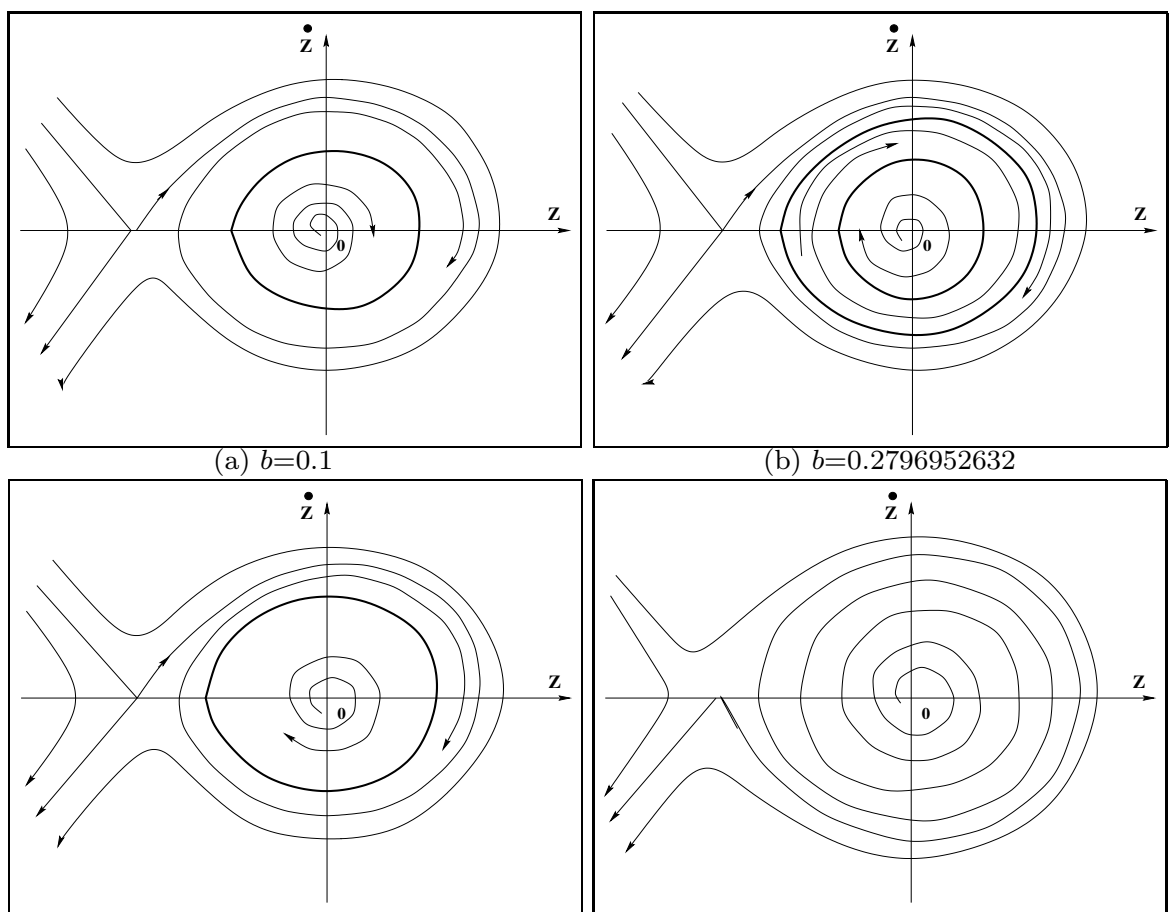

(c) $b=0.2797061632$

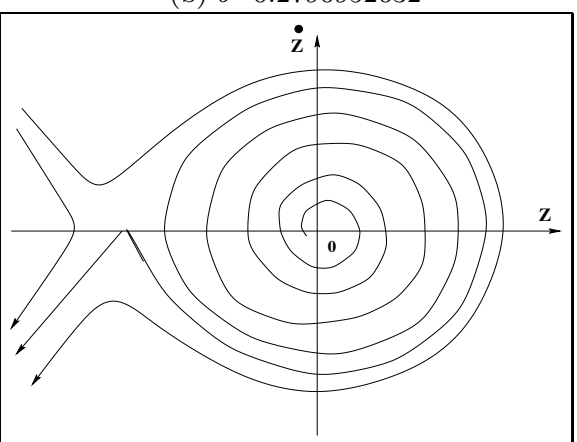

(d) $b=0.29$

FIG. 4.2. Sketch of the trajectories of system (3.25) in the phase plane for several values of $b$.

$$
=\frac{1}{2} X_{1}^{2}+\frac{1}{3} b X_{1}^{3}+\frac{1}{2} X_{2}^{2}+\epsilon\left\{-\tilde{I}_{1}\left(1-\frac{\tilde{I_{2}}}{\tilde{I}_{1}}\right)\right\},
$$

where

$$
\begin{aligned}
& \tilde{I}_{1}=\int_{X_{1}(0)}^{X_{1}(t)}\left(2 k_{1}-r^{2}-\frac{2}{3} b r^{3}\right)^{\frac{1}{2}} d r \\
& \tilde{I}_{2}=\frac{1}{3} \int_{X_{1}(0)}^{X_{1}(t)}\left(2 k_{1}-r^{2}-\frac{2}{3} b r^{3}\right)^{\frac{3}{2}} d r .
\end{aligned}
$$

In the $\left(X_{1}, X_{2}\right)$-phase plane we now define the positive $X_{1}$-axis to be a Poincare section. At $t=0$ we start in $\left(X_{1}(0), 0\right)$ with $X_{1}(0)>0$. After some time $\left(t=t_{0}\right)$ we return to the Poincare section, that is, $\left(X_{1}\left(t_{0}\right), X_{2}\left(t_{0}\right)\right)=\left(X_{1}\left(t_{0}\right), 0\right)$ with $X_{1}\left(t_{0}\right)>0$. From (4.11) and (4.19), we know that

$$
\frac{1}{2} X_{1}^{2}(t)+\frac{1}{3} b X_{1}^{3}(t)+\frac{1}{2} X_{2}^{2}(t)=k^{*}+\epsilon\left(I_{1}\left(1-\frac{I_{2}}{I_{1}}\right)\right)+\mathcal{O}\left(\epsilon^{2}\right) .
$$

For a periodic solution $Q=\frac{I_{2}}{I_{1}}$ should be 1 , and (4.21) then becomes

$$
\frac{1}{2} X_{1}^{2}(t)+\frac{1}{3} b X_{1}^{3}(t)+\frac{1}{2} X_{2}^{2}(t)=k^{*}+\mathcal{O}\left(\epsilon^{2}\right),
$$

where $k^{*}$ is assumed to be the constant in the approximation of a first integral for which a periodic solution exists. From the numerical calculations of $Q=\frac{I_{2}}{I_{1}}$ (see also Figure 4.1) it follows that there are two possibilities: 
(i) $\frac{I_{2}}{I_{1}}<1$ for $k_{1}<k^{*}$ and $\frac{I_{2}}{I_{1}}>1$ for $k_{1}>k^{*}$, or

(ii) $\frac{I_{2}}{I_{1}}>1$ for $k_{1}<k^{*}$ and $\frac{I_{2}}{I_{1}}<1$ for $k_{1}>k^{*}$.

In the first case (i) it follows from (4.21) that if $\frac{I_{2}}{I_{1}}<1$ for $k_{1}<k^{*}$ then $\epsilon\left(2 I_{1}\left(1-\frac{I_{2}}{I_{1}}\right)\right)>$ 0 , and so $X_{1}\left(t_{0}\right)>X_{1}(0)$. And similarly if $\frac{I_{2}}{I_{1}}>1$ for $k_{1}>k^{*}$ then $\epsilon\left(2 I_{1}\left(1-\frac{I_{2}}{I_{1}}\right)\right)<$ 0 , and so $X_{1}\left(t_{0}\right)<X_{1}(0)$. So in the first case (i) the periodic solution is stable. In case (ii) it can be shown similarly that the periodic solution is unstable. The period $T$ of the periodic solution can be determined from

$$
\frac{d X_{1}}{d t}=X_{2}=\sqrt{\left(2 k^{*}-X_{1}^{2}-\frac{2}{3} b X_{1}^{3}\right)},
$$

or from

$$
T=\int_{A}^{B} \frac{2 d X_{1}}{\sqrt{\left(2 k^{*}-X_{1}^{2}-\frac{2}{3} b X_{1}^{3}\right)}},
$$

where $-\frac{1}{b}<A<B<\frac{1}{2 b}$ and where $A, B$ satisfy $\frac{1}{2} X_{1}^{2}+\frac{1}{3} b X_{1}^{3}=k^{*}$. It should be observed that if $k^{*} \rightarrow \frac{1}{6 b^{2}}$ then $A \downarrow-\frac{1}{b}$ and $B \uparrow \frac{1}{2 b}$ so

$$
\begin{aligned}
T & =\lim _{k^{*} \rightarrow \frac{1}{6 b^{2}}} \int_{A}^{B} \frac{2 d X_{1}}{\sqrt{\left(2 k^{*}-X_{1}^{2}-\frac{2}{3} b X_{1}^{3}\right)}} \\
& =\int_{-\frac{1}{b}}^{\frac{1}{2 b}} \frac{2 d X_{1}}{\left(X_{1}+\frac{1}{b}\right)\left(-\frac{2}{3} X_{1}+\frac{1}{3}\right)^{\frac{1}{2}}} \\
& =\left[-4 \tanh ^{-1}\left(-\frac{2}{3} b r+\frac{1}{3}\right)^{\frac{1}{2}}\right]_{-\frac{1}{b}}^{\frac{1}{2 b}}=\infty
\end{aligned}
$$

Using (4.24) we have determined $T(b)$ numerically (see Figure 4.3).

5. Conclusions and remarks. In this paper it has been shown that the perturbation method based on integrating factors can be used efficiently to approximate first integrals for a generalized Rayleigh oscillator. The method can also be applied to other nonlinear oscillator equations that are integrable when the small parameter is zero. In section 2 (and 3) of this paper an asymptotic justification of the presented perturbation method has been given. For a generalized Rayleigh oscillator it has been shown how the existence and stability of time-periodic solutions can be deduced from the approximations of the first integrals. For the nonlinear Rayleigh oscillator it has been shown that there exists one stable periodic solution if $0<b<\sqrt{\frac{6}{77}}+\mathcal{O}\left(\epsilon^{2}\right)$, that there are two periodic solutions (one stable and one unstable) if $\sqrt{\frac{6}{77}}+\mathcal{O}\left(\epsilon^{2}\right) \leq b \leq \sqrt{\frac{6}{77}}+\delta\left(\delta\right.$ is approximately $\left.1.09 \times 10^{-5}\right)$, and that there are no periodic solutions if $b>\sqrt{\frac{6}{77}}+\delta$.

Acknowledgement-This research project was sponsored by Secondary Teacher Development Programme (PGSM)(Indonesia) and The University of Technology in Delft (The Netherlands). 


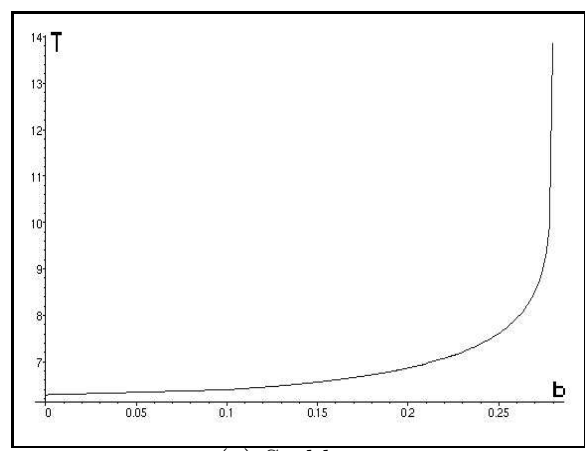

(a) Stable

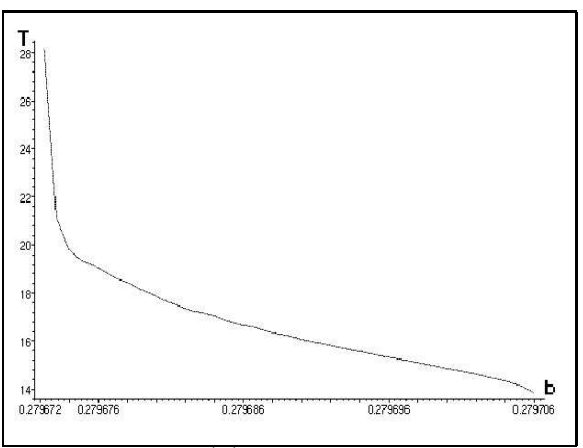

(b) Unstable

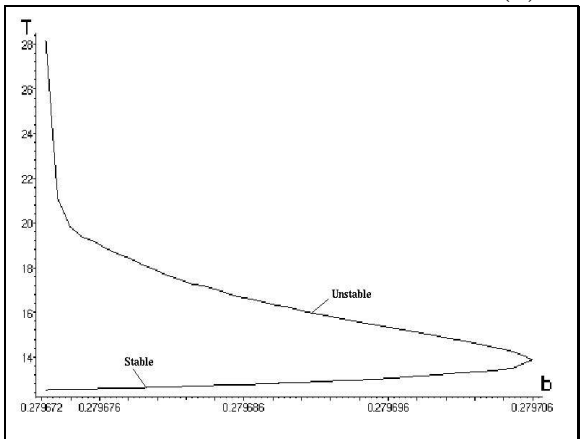

(d) Stable and Unstable

FIG. 4.3. Plot of the period $T$ of the stable and unstable periodic solutions as functions of $b$.

\section{REFERENCES}

[1] A. Doelman and F. Verhulst, Bifurcations of Strongly Non-linear Self-excited Oscillations, Mathematical Methods in the Applied Sciences, Vol. 17, 1994, pp. 189-207.

[2] A.H. Nayfeh and D.T. Mook, Nonlinear Oscillations, John Wiley \& Sons, New York, 1985.

[3] C.G.A. van der Beek, Normal form and periodic solutions in the theory of non-linear oscillations. Existence and asymptotic theory, International journal of non-linear mechanics, Vol. 24, No. 4, 1989, pp. 263-279.

[4] F. Verhulst, Nonlinear differential equations and dynamical systems, Springer-Verlag, Berlin, 1996.

[5] J. Guckenheimer and P. Holmes, Nonlinear Oscillations, Dynamical Systems, and Bifurcation of Vector Fields, Applied Math. Sc.42, Springer-Verlag, New York, 1983.

[6] J. Kevorkian and J.D. Cole, Multiple Scale and Singular Perturbation Methods, Applied Math. Sc.114, Springer-Verlag, New York, 1996.

[7] S. Wiggins, Introduction to Applied Nonlinear Dynamical Systems and Chaos, Springer-Verlag, New York, 1990.

[8] W.T. van Horssen and J.W. Reyn, Bifurcation of limit cycles in a particular class of quadratic systems, Differential and integral Equations, Vol. 8, No. 4, 1995, pp. 907-920.

[9] W.T. van Horssen, On Integrating Factors for Ordinary Differential Equations, Nieuw Archief voor Wiskunde, Vol. 15, 1997, pp. 15-26.

[10] W.T. van Horssen, A Perturbation Method Based on Integrating Factors, SIAM journal on Applied Mathematics, Vol. 59, No. 4, 1999, pp. 1427-1443.

[11] W.T. van Horssen, A Perturbation Method Based on Integrating Vectors and Multiple Scales, SIAM journal on Applied Mathematics, Vol. 59, No. 4, 1999, pp. 1444-1467. 